In the analogous cases of hypermetropia why should we pay so much attention to the latent error, going even to the point of paralysing the ciliary muscle to determine it, if at the same time we ignore the latent muscle imbalance? There can remain no doubt of the intimate relationship between accommodation and convergence, and cases of asthenopia cannot be completely understood without the fullest possible knowledge of each.

As a matter of fact failure to measure muscle imbalance adequately is the commonest cause of failure, in the experience of the writer, to relieve asthenopia.

\title{
FACTORS DETERMINING THE ADVISABILITY OF SELECTIVE OPHTHALMIC SURGERY
}

\author{
BY \\ L. Webster Fox, M.D., LL.D., Philadelphia \\ PROFESSOR OF OPHTHALMOLOGY, GRADUATE SCHOOL OF MEDICINE, \\ UNIVERSITY OF PENNSYLVANIA
}

THE field of ophthalmic surgery, while very comprehensive, may for the purpose of this discussion be narrowed down to those procedures indicated for the relief of the troublesome affections of declining years, especially cataract and glaucoma. A great tendency exists to regard these conditions as individual as well as independent affections, only partially related to the complex whole of the human organism. In fact the preparation of the patient and the field of operation are very often considered only as regards the possibility of infection of the field, or of the blood through the field of operation.

Ocular, or in fact any disease in the declining decades of life may be influenced by the same factors that have influenced the patient's reactions to the vicissitudes of life in general. The man who never acknowledges defeat, nearly always shows a physical resistance out of all proportion to his obvious physical equipment. The laboratory men working along the line of microscopic organisms never seem to have adequately explained, from a biological standpoint, the phenomena of individual resistance to disease, but have complicated our possible understanding of it, from a clinical point of view, by references to speculations involving the use of new and complicated expressions. Thus for our own understanding we are compelled to fall back upon the homely expression "individual resistance to disease," something every practitioner can readily appreciate. 
The ease with which the skilful operator can attain his objective in the ocular surgical conditions of declining years, may mislead one into believing there is no shock connected with these procedures. This is an error! The surgical treatment of cataract and glaucoma are major undertakings. Almost any time during the operation, or in the course of the convalescence, neurotic manifestations may supervene. Mental disturbances, while always possible, are now less frequent than formerly, due to a better appreciation of the factors contributing to them.

An analysis of the patient's stamina and his reactions to things in general, as well as to his environmental influences will go far towards determining whether or not he will constitute a good operative risk. No little has been written concerning the influence of the teeth, the tonsils, the sinuses, the prostate, the heart, the liver, the kidneys, the blood-pressure, the blood-sugar, etc., etc., yet every surgeon of experience can point to most remarkable success in patients whose clinical and laboratory record was anything but enviable. Too much reliance upon these factors is certain to deprive a great many deserving persons of the assistance which ocular surgery can afford. On the other hand, disaster will happen, at times, to patients with a clear bill of health in this regard, and in whom the operation has been performed with consummate skill.

The surgeon of experience may frequently ascertain these determining factors by his own powers of observation independent of outside aid, but certain it is our laboratory aids are not so effectual in this regard as we could desire them to be. The diverse contact with human beings which a surgeon of large experience has, frequently enables him to grasp quickly the outstanding features of any patient's personal equation; that he can anticipate uninterrupted convalescence with this patient, and on the other hand difficulties and complications otherwise unexpected.

Unfortunately the possession of such a rare sense does not always carry with it the judgment to exercise it continuously, and we find surprising untoward happenings where a moment's hesitation might have enabled us to take advantage of the warnings given of dangers ahead. The busy man is likely to be taken off his guard by extenuating circumstances and to avoid this it is essential that he provide himself with certain working rules, varying with different men, but adapted to the exigencies of his own surroundings.

Keeping a patient under observation as an ambulant or office patient over as long a period as possible will familiarize the patient with the doctor and his manner as well as provide the doctor with a means of gauging the calibre of the patient. Patients so handled provide the very best operative risks even in the face of serious 
operative difficulties. The patient's reactions to the medicaments we employ, also indicate the functional ability of the eye. Many an operation has been ruined by faulty anaesthesia-a high tension eye preventing the absorption of the cocaine solution.

There is no doubt as to the disastrous influence of inflammatory conditions of the lacrimo-nasal canal, the upper air-passages and adjacent sinuses, but every case of epiphora should not be regarded as evidence of a focus of infection in the lacrimo-nasal tract. A patient with such a complication may under observation and treatment be brought to a stage where the secretion in which the eye is bathed is practically sterile.' Such an eye may, if bandaged for several days show a positive culture of some of the pus-producing micro-organisms. Should this disappear under treatment within a few days, it does not indicate that an operation involving the opening of the eye should be abandoned, nor does it point to the justification for the handling of the patient in the routine manner. Rather does it demonstrate the necessity of opening the dressing of that eye, no matter what the operation, just as soon as is consistent with the security for the objective of the operation. In those communities where the uncomplicated cases are the exception, it will be noted that the surgeons favour as little occlusive dressing as possible and as short a time for the same as consistent with safety, and some none at all, placing a cage over the eye for its protection. These judgments are not inspired by caprice but by experience.

Patients in the cataract and glaucoma period of life also belong to the epoch when the vital organs are beginning to show the stress of the years that have gone before. Hence, we find cardiovascular affections, Bright's disease, arteriosclerosis, diabetes, etc., to add to our difficulties. In referring to these for the benefit of the attending physician it is well to mention all the synonyms, since many men get themselves into the habit of using one name to interpret their understanding of a sequence or cycle of disorders.

Diabetes as represented by glycosuria has done much to disturb our ophthalmic equanimity in the past. Our practice has been to operate in all such patients provided the glycosuria did not exceed 3 per cent. Obviously patients showing more than that were disturbed more by their diabetes than by their ocular condition, and were not likely to present themselves for surgical consideration.

We have been assured by Dr. Orlando Petty, that although we have been within safe confines by this ruling, our approach to the subject has been wrong and that in the light of the recent work in this field we should regard the condition in terms of blood sugar. Thus blood sugar up to 170 will tolerate ophthalmic operations. Also under proper control and proper insulin treatment, any case of hyperglycaemia can be reduced to operative level within a few 
hours and maintained there. This is now quite a commonplace accomplishment.

We are inclined to regard the association of diabetes with detrimental conditions of the eye as somewhat exaggerated. Out of 254 patients under control in the diabetic clinic of the Graduate Hospital of the University of Pennsylvania, at least 141 showed no eye conditions. In some 27 cases there was only a simple choroiditis most commonly situated at the temporal side of the disc. In only six cases were haemorrhages noted. In 17 a combination of choroiditis and haemorrhages was observed. Cataract occurred in 19 cases. Two cases of choked disc were seen which subsided under diabetic treatment. While these cases occurred among diabetics, it is difficult to assume that the relationship between the eye conditions and the diabetes was positive. In the younger diabetics, the eye troubles were very few, showing that age is still the great factor.

The popularity of high blood-pressure as a factor in determining all expressions of human activity cannot be entirely ignored from an academic standpoint, although from a practical standpoint it may go far towards eliminating our surgery if we take it too seriously. Unless haemorrhages have occurred within the eye to the extent that vision is already irreparably ruined it is not to be considered as a contra-indication for operation upon the eye. Our technique in cataract operations includes preliminary canthotomy and the degree of haemorrhage following this may be taken as' an index of the bleeding possibilities when the globe is subjected to operation. This conclusion does not imply that treatment directed towards the high blood-pressure should be withheld.

The functional activity of the kidney is of more concern to us than the structural lesions determined by urinalysis. The quantity eliminated in 24 hours is more important than the mere presence of albumen and casts. Also the presence or absence of those elements concerned with cystitis and enlarged prostate are of great significance. A prostatic patient by reason of his inability to pass his water lying down may add a very disastrous feature to our work. This condition should be determined before admission to the hospital so that provision can be made for it. In an uneventful case there is no great objection to such a patient using the commode six hours after operation. Catheterization may be employed before that time although it is seldom necessary if the patient has been instructed to use the toilet in the ordinary manner prior to the operation. However if such a patient harbours the impression that he is restrained and must keep flat on his back for 24 hours, or until the next visit of the doctor, one need not be surprised if retention of urine, vesical spasm, and mild delirium supervene. The 
normal routine of these patients must be disturbed as little as possible, and it is surprising how little it need be.

The determination of the proper time for ophthalmic operations in persons in the declining years of life entails the exercise of considerable judgment, but one must be careful not to err on the too conservative side of the situation lest visual benefit be denied to those who have little else to ease their way along.

\title{
A NOTE ON THE HYDROGEN ION CONCENTRATION OF THE VITREOUS IN EYES AFFECTED BY NAPHTHALENE
}

\author{
BY \\ Dorothy R. Adams and Phyllis Tookey Kerridge \\ UNIVERSITY COLLEGE, LONDON
}

THE second stage of the development of naphthalene cataract in rabbits, is an opacity which begins in the superficial layers of the posterior cortex of the lens, i.e., in the region adjacent to the vitreous. It has been shown (Adams, 1930) that the cataract is almost invariably preceded by the formation of extensive retinal exudates, an oedematous change which might easily affect the vitreous in such a way as to alter its hydrogen ion concentration. Experiments on the isolated lens have demonstrated its sensitivity to the $\mathrm{cH}$ of the surrounding medium. It is known, for instance, that a superficial opacity can readily be produced by immersing a lens in an acid solution, and that by using solutions of different $\mathrm{cH}$ values, one can determine approximately the isoelectric point of the lens (Gullotta, 1926). It was therefore considered worth while to investigate the $\mathrm{cH}$ of the vitreous in rabbits at different times after the administration of naphthalene, in order to see whether it exhibited any change in hydrogen ion concentration which might account for the local condition of the lens, or could be correlated with the oedematous changes in the retina. At the same time it was interesting to find the hydrogen ion concentration of normal vitreous, since it has not been determined previously for the rabbit.

Experimental.-In describing these experiments* it is realised that the values obtained for the hydrogen ion concentration of the

\footnotetext{
*These experiments formed part of the research work for the Committee of Vision
} of the Medical Research Council. 